\title{
LA ESCUELA COMO ELEMENTO INTEGRADOR: ESTUDIO DE LA ESO EN NAVARRA
}

\section{SCHOOL AS AN INTEGRATOR: SECONDARY'S STUDIES IN NAVARRA}

\author{
Neus Caparrós Civera \\ Universidad de La Rioja. España/Spain \\ caparros@unirioja.es \\ Esther Casares García \\ Universidad Pública de Navarra. España/Spain \\ casares@unavarra.es
}

Recibido/Received: 27/03/2014

Modificado/Modified: 30/05/2014

Aceptado/Accepted: 27/06/2014

\section{RESUMEN}

El artículo que aquí se presenta recoge el trabajo realizado en los centros educativos de enseñanza secundaria en la Comunidad Foral de Navarra. El objetivo de la investigación era analizar el fenómeno de la inmigración a través de la percepción de los diferentes agentes implicados en el propio proceso: alumnado, docentes y otros profesionales. La metodología utilizada ha sido de corte cualitativo, basado en entrevistas en profundidad a jefes de estudios de centros escolares con un alto índice de presencia de alumnos inmigrantes y, de corte cuantitativo basado en más de 800 encuestas a los alumnos de dichos centros repartidos en la geografía navarra. Los resultados obtenidos en el estudio podrían ser considerados como punto de partida para el diseño de futuras políticas sociales para favorecer la integración del inmigrante, sobre todo durante la adolescencia y para romper prejuicios entre autóctonos y extranjeros y de los extranjeros entre sí.

\section{PALABRAS CLAVE}

Inmigración, integración, educación secundaria, adolescencia, subcultura, políticas sociales, escuela.

\section{SUMARIO}

1. Planteamiento. 2. Metodología. 3. Resultados. 4. Conclusiones. Bibliografía.

\begin{abstract}
The article presented here reflects the work done in schools of secondary education in Navarre. The objective of the research was to analyze the phenomenon of immigration through the perception of the different actors involved in the process itself: students, teachers and other professionals. The methodology is qualitative, based on interviews in depth to chiefs of studies of centers school by a high index of presence of immigrant pupils and, and quantitative based in more than 800 surveys to the pupils of the above mentioned centers distributed in the Navarre geography. The results obtained in it will be considered to be an starting point of item for the design of future social policies, to improve the integration of the immigrant, especially during the adolescence and to break prejudices of the native ones towards the immigrants, of these towards the autochthonous ones, and also among the foreigners.
\end{abstract}

\section{KEYWORDS}

Immigration, integration, secondary education, adolescence, subculture, social policies, school. 


\section{CONTENTS}

1. Approach. 2. Methodology. 3. Results. 4. Conclusions. References.

\section{PLANTEAMiento}

El estudio de la realidad navarra en relación al fenómeno de la inmigración pone de manifiesto, entre otros temas, que en los últimos años hayan llegado a esta comunidad un mayor número de personas en busca de un futuro mejor y, en consecuencia, haya crecido, de forma notable, el número de alumnos inmigrantes que se incorporan al sistema educativo.

Los inmigrantes se desplazan en busca de una vida mejor, una tierra diferente, un espacio distinto. Pero la nueva dimensión no conlleva la pérdida de sus hábitos, costumbres y maneras de expresarse y convivir, puesto que seguirán manifestándose y comportándose como si de su tierra se tratase.

Esta situación afecta a todas las generaciones de las familias desplazadas, tanto mayores como pequeños, menores que, si están en edad escolar, deberán asistir a un centro educativo, tal como lo marcan las leyes del país receptor.

Según las cifras utilizadas por el Consejo Escolar de Navarra referidas al año de realización del presente estudio, (2009-2010), el número de escolares inmigrantes fue de 11.847 , un $11,5 \%$ del total de escolares matriculados en el sistema educativo navarro, habiéndose registrado un incremento del $37 \%$ respecto a cursos anteriores. Navarra es una de las comunidades con mayor presencia porcentual de alumnos inmigrantes en sus aulas, tras La Rioja, Madrid y Cataluña.

En más de cuarenta centros públicos el porcentaje de alumnos inmigrantes supera el $25 \%$, y en la mitad de estos, el $40 \%$; y en ocho se alcanza el $80 \%$. Para diversos responsables educativos el límite de alumnos para que se pueda llevar a cabo una buena integración no debería superar el 30\%.

Los alumnos proceden de diferentes países, con la consecuente expresión de diversas realidades de origen, produciéndose en muchas ocasiones más una adaptación que no una integración, se acostumbran a la nueva realidad, conviven con ella lo que no significa que exista una plena integración al medio, que se les hace, en muchas ocasiones, hostil. A través de los nuevos alumnos, en las aulas de los centros educativos se entra en contacto con otras culturas, lo que puede ocasionar el intercambio de valores, de los diferentes valores de las culturas de cada alumno y alumna, aunque en muchas ocasiones no se consiga de la manera esperada.

Educar a todo el alumnado es la pretensión de la Educación, sin distinguir la procedencia o el origen de cada uno de los estudiantes de los que se compone un aula. Pero este objetivo no se cumple en algunos casos y la inmigración en algunos casos da cuenta de ello, porque existen problemas de integración en las aulas que dificultan cualquier aprendizaje de los recién llegados, de los diferentes, de "los otros", diferentes muchas veces solo por el hecho de proceder de otro territorio.

Migrar, dejar el espacio natal para pasar a residir en otro de manera temporal o permanente forma parte del comportamiento natural de las sociedades humanas (Besalú, 2002) por lo que los seres humanos se ven sometidos de manera constante a adaptaciones en los nuevos territorios de asentamiento. En este sentido los centros escolares juegan un papel muy importante, puesto que es el espacio en el que van a aprender a desenvolverse los hijos 
de los recién llegados, y es el espacio en el que van a interiorizar normas y pautas de comportamiento de la sociedad receptora.

Para conseguir una buena integración socio-educativa de estos alumnos deben superarse una serie de dificultades que se presentan como barreras. En ocasiones los nuevos alumnos no hablan castellano, pertenecen a distintos grupos culturales y tienen otras costumbres y creencias religiosas. Estas características demandan un esfuerzo notable para conseguir que la integración sea efectiva, por parte de las Administraciones Públicas y de todos los representantes de la comunidad educativa (alumnos, padres y madres, profesores...).

En diferentes informes y estudios realizados en otros países también se pone de relieve que la presencia de alumnos de origen inmigrante puede ser un factor de desequilibrio en las escuelas (Ponce, 2007; Aja-Arango, 2006). Por otro lado en realidades cercanas a la nuestra, como es el caso de Francia, se constata que muchos jóvenes inmigrantes tanto si han inmigrado con sus padres o pertenecen a la primera generación nacida en el país de destino, no se integran en la sociedad que los acoge, presentando serios problemas de comportamiento, tanto individual como colectivo, sobre todo a partir de la adolescencia (Prevost, 2004).

La no integración o también llamada no socialización de los jóvenes puede considerarse como el resultado de un proceso complejo en el que convergen múltiples causas, siendo algunas de ellas, entre otras, la incorporación al sistema escolar con niveles educativos inferiores a la media de los jóvenes de su edad del país receptor; Problemas con el idioma; Valores, sentimientos y creencias de la sociedad receptora distintas a la socialización recibida en la familia; Métodos docentes y relaciones con los profesores diferentes, incluso contradictorios a los existentes en los países de origen; rechazo y distanciamiento con los estudiantes del centro por el diferente color de la piel, por sus rasgos físicos o por su forma de hablar y proyectos formativos que no consiguen los objetivos propuestos por el centro para la integración del alumnado extranjero en este campo (Valero, 2002; Barbosa, 2007).

En los últimos tiempos se han realizado diversos estudios sobre la interculturalidad en el entorno escolar, pero en muchas de estas ocasiones lo que se ha tenido en cuenta es el punto de vista del profesorado, qué se espera de él, en la manera de realizar actividades dentro y fuera del aula y de las actitudes conseguidas con ellas. (García Castaño y otros, 2008) sin tener en cuenta otras percepciones como son los propios implicados, alumnos autóctonos y alumnos extranjeros. También cabe destacar que a nivel europeo las investigaciones que se han realizado son principalmente de corte cuantitativo, dejando de lado el enfoque cualitativo por no gozar del mismo reconocimiento, no obstante la realización de un estudio en profundidad requiere de las dos perspectivas (cuantitativa y cualitativa) conjugando los dos enfoques metodológicos.

Si consideramos que los centros educativos constituyen el primer contacto de los alumnos y las alumnas inmigrantes con la sociedad acogedora, deberemos tenerlos en consideración al realizar cualquier estudio sobre la interculturalidad. Los centros son el primer referente, para los extranjeros, en cuanto a sus posibilidades de aceptación, de reconocimiento y de su capacidad para formar parte o no, como un miembro más, de una sociedad distinta de aquella que tuvieron que abandonar, con todas las consecuencias positivas y negativas que ello acarrea.

Los equipos directivos y docentes de los centros tienen un papel principal para promover acciones que favorezcan el ajuste entre los diferentes alumnos que tengan. Y también podrán fomentar la colaboración de las propias familias para que no se queden al margen de la actividad tanto escolar como extraescolar que se origine. 
En este sentido el estudio que a continuación se describe pretende, en primer lugar, poner de manifiesto cuál es la percepción que tienen los alumnos entre sí, tanto los extranjeros como los nativos en el proceso de integración educativa, las dificultades y apoyos que encuentran, la opinión de los profesores, de los educadores, la actitud de las familias, la identificación de posibles conflictos y otras situaciones que se puedan manifestar y en segundo lugar pretende ser una de las bases en las que se puedan asentar nuevas políticas de intervención en materia de integración de niños y jóvenes procedentes de otros países.

\section{METODOLOGÍA DE LA INVESTIGACIÓN}

Para acometer el objeto de estudio se elaboraron una serie de hipótesis como punto de partida, tales como que: Los inmigrantes se han ido identificando a sí mismos como alumnos distintos, cuando no rechazados y que para sobrevivir en una tierra extraña han tenido que refugiarse en sus propios grupos étnicos donde se han visto acogidos en condiciones de igualdad.

Una situación de "excluidos" latentes puede provocar la aparición de procesos de rechazo y de agresión contra la sociedad receptora, por parte de aquellos que no se sienten en la misma igualdad de condiciones que las personas autóctonas, agresiones que pueden tener numerosas expresiones, tales como agresiones a compañeros de los otros grupos; indisciplina en los centros, tanto en horas de clase como fuera de ellas; no querer adaptarse al centro y acatar sus normas, llegando incluso a formar pequeñas bandas que puedan recurrir a la violencia en determinados momentos, atentando contra compañeros de clase o contra el propio centro, ocasionando desperfectos.

Por tanto, con el fin de mejorar la integración de los inmigrantes en los centros escolares, para facilitar la convivencia y mejorar los procesos de adaptación y aceptación, es necesario conocer en qué grado existen procesos de exclusión y rechazo hacia estos jóvenes, teniendo en cuenta los factores que puedan provocar estas actitudes (Torreblanca, 2006).

Si hablamos de exclusión deberemos hablar también de inclusión (Karsz, 2000), puesto que ambos conceptos forman parte de un continuo indisociable en el que intervienen las mismas variables. Los dos son conceptos opuestos, pero se van construyendo y reconstruyendo a la par. Podemos decir que son las prácticas y actitudes del propio contexto social las que generan las barreras que obstaculizan o las ayudas que favorecen el acceso y la participación de las personas, en nuestro caso niños y adolescentes en edad escolar.

Para llevar a cabo el estudio fue necesario abordarlo desde diferentes perspectivas: la de los adolescentes, la de los centros docentes, instituciones y organizaciones, y la de los expertos y otros agentes sociales como trabajadores sociales y educadores sociales. También se tuvieron en cuenta estudios realizados anteriormente sobre el tema objeto de estudio analizando de manera exhaustiva toda la información localizada.

Todo ello fue abordado tanto de manera cuantitativa, a partir de encuestas a los propios alumnos de los centros escolares de formación secundaria en Navarra, analizadas con el programa SPSS, como de manera cualitativa, a través de entrevistas en profundidad a la población diana del estudio y a los agentes sociales implicados. La propuesta era profundizar e indagar en el imaginario social de los inmigrantes y de los autóctonos en la realidad concreta de los centros de enseñanza secundaria obligatoria.

Para analizar la situación de los inmigrantes se trabajó con una muestra selectiva, porque se seleccionaron todos los centros públicos y concertados donde los alumnos inmigrantes representasen el $15 \% \mathrm{y}$, por tanto, donde mejor se pudieran analizar los problemas a los que 
se debían enfrentar los inmigrantes, pero también se realizó un estudio de contraste a tres centros concertados y dos públicos, en los que hubiera poca presencia de alumnado inmigrante, para "contrastar" con los otros centros la actitud de los alumnos respecto al tema de la inmigración y si las opiniones respecto a ellos diferían en si al estar en contacto o no con ellos en las aulas.

\section{RESULTADOS}

\subsection{La perspectiva de los estudiantes inmigrantes}

Los inmigrantes son los protagonistas del proceso social de integración y quienes viven el proceso a partir de experiencias tanto positivas como negativas. Por tanto, el estudio los ha tenido en cuenta para conocer cómo viven su experiencia entre los miembros de la sociedad receptora, y para conocer en qué grado se sienten acogidos o rechazados por los nativos. Pero también ha sido de especial interés saber la opinión de la otra parte la de los jóvenes autóctonos receptores de sus homólogos extranjeros, para saber cuál es su posición al respecto.

Del análisis de los resultados obtenidos se desprende que los colectivos de inmigrantes forman una nueva clase social difusa. Socialmente son grupos poco visibles, dispersos en los barrios de las ciudades, pero con momentos y lugares de encuentro que fortalecen su identidad y sus lazos de cohesión social.

La dispersión familiar, en el caso de los hispanoamericanos, es frecuente. Social y educativamente la estructura familiar influye de forma determinante en los adolescentes. Algo más de la mitad (55,2\%) viven con sus padres y un $27,3 \%$ con el padre o la madre. Y de los encuestados más del $69,0 \%$ son procedentes de Europa del Este y viven con los padres y un $13,8 \%$ con el padre o la madre.

Los alumnos inmigrantes tienen baja identificación con España. Solamente el $45.1 \%$ de los hispanoamericanos desearía vivir cuando sea mayor en este país, mientras que a uno de cada cuatro le gustaría vivir en otro país diferente de España incluso del suyo propio. En el caso de los alumnos de países del Este, el 58,6\%, les gustaría vivir aquí y un 31,0\% volver a su país de origen.

En cuanto a las relaciones con compañeros en el centro el 78,5\% de los alumnos navarros se relaciona con sus iguales, tanto navarros como de otras comunidades. En el caso de los hispanoamericanos hay un $26,7 \%$ que se relaciona con navarros y un $49,6 \%$ que se relaciona con todos. Un $20,7 \%$ se relaciona con compañeros de su país o de otros países y no parece que los navarros estén muy abiertos a compañeros de países extranjeros, mientras que sí lo están los alumnos procedentes de otras nacionalidades.

Fuera del horario escolar se incrementa la distancia entre navarros e inmigrantes. Por tanto, se construyen, en alguna medida, dos mundos juveniles paralelos con pocas posibilidades de convergencia y muchos navarros, fuera del centro escolar, se hacen más impermeables a la presencia de inmigrantes en sus grupos de amigos para el disfrute del tiempo libre.

Los planteamientos de los alumnos al terminar los estudios en el centro escolar presentan diferencias muy notables en la manera de definir su futuro al concluirlos. Alrededor del $60 \%$ de los alumnos de los países del Este y los navarros, se inclinan por los estudios universitarios. El 41,3\% de los hispanoamericanos también piensan en la universidad y el 
$30,1 \%$ en realizar cursos de formación profesional, mientras que los magrebíes mayoritariamente se inclinan por acceder al mercado laboral.

Tabla 1: Posibilidades de estudios superiores al finalizar la educación secundaria, según el origen

\begin{tabular}{|l|l|l|l|l|}
\hline \multirow{2}{*}{} & \multicolumn{3}{|l|}{ Origen } & \multicolumn{2}{l|}{} \\
\cline { 2 - 5 } & España & Hispanoamérica & Europa del Este & Magrebíes \\
\hline Trabajar & $5,8 \%$ & $14,0 \%$ & $6,9 \%$ & $35,7 \%$ \\
\hline Ir a la universidad & $59,0 \%$ & $41,3 \%$ & $62,1 \%$ & $14,3 \%$ \\
\hline Cursos de formación & $26,4 \%$ & $30,1 \%$ & $20,7 \%$ & $28,6 \%$ \\
\hline Ir a otro país & $0,7 \%$ & $5,6 \%$ &, $0 \%$ & $7,1 \%$ \\
\hline Nada/ no lo sé & $8,2 \%$ & $9,1 \%$ & $10,3 \%$ & $14,3 \%$ \\
\hline
\end{tabular}

Fuente: Elaboración propia a partir de los datos del estudio

El rechazo hacia los inmigrantes no existe de manera generalizada puesto que los resultados ponen de manifiesto que solamente 42 alumnos $(6,3 \%)$ se han sentido aislados o rechazados. No obstante hay 42 casos de rechazo que conviene analizar más detalladamente, ya que solamente $11,2 \%$ corresponde a alumnos españoles. El resto son todos extranjeros. Un análisis más profundo del rechazo nos ha permitido detectar que en todos los colectivos es algo mayor el número de alumnas que se sienten rechazadas que el de alumnos. Análisis que debería realizarse más en profundidad teniendo en cuenta la perspectiva de género.

Tabla 2: Actividades extraescolares según el origen

\begin{tabular}{|c|c|c|c|c|c|}
\hline & \multicolumn{4}{|c|}{ Origen } \\
\hline & & España & Hispanoamérica & Europa del Este & Magreb \\
\hline \multirow[t]{4}{*}{ A diario } & Recuento & 44 & 3 & 1 & 0 \\
\hline & $\begin{array}{l}\% \text { dentro de Qué haces en tu } \\
\text { tiempo libre. Actividades } \\
\text { extraescolares }\end{array}$ & $91,7 \%$ & $6,3 \%$ & $2,1 \%$ &, $0 \%$ \\
\hline & $\%$ dentro de Origen & $9,7 \%$ & $2,1 \%$ & $3,4 \%$ &, $0 \%$ \\
\hline & $\%$ del total & $6,9 \%$ &, $5 \%$ &, $2 \%$ &, $0 \%$ \\
\hline \multirow{4}{*}{$\begin{array}{l}\text { Algunos } \\
\text { días }\end{array}$} & Recuento & 160 & 29 & 5 & 4 \\
\hline & $\begin{array}{l}\% \text { dentro de Qué haces en tu } \\
\text { tiempo libre. Actividades } \\
\text { extraescolares }\end{array}$ & $80,8 \%$ & $14,6 \%$ & $2,5 \%$ & $2,0 \%$ \\
\hline & $\%$ dentro de Origen & $35,3 \%$ & $20,3 \%$ & $17,2 \%$ & $28,6 \%$ \\
\hline & $\%$ del total & $25,0 \%$ & $4,5 \%$ &, $8 \%$ &, $6 \%$ \\
\hline \multirow{4}{*}{$\begin{array}{l}\text { De vez } \\
\text { en } \\
\text { cuando }\end{array}$} & Recuento & 62 & 52 & 12 & 2 \\
\hline & $\begin{array}{l}\text { \% dentro de Qué haces en tu } \\
\text { tiempo libre. Actividades } \\
\text { extraescolares }\end{array}$ & $48,4 \%$ & $40,6 \%$ & $9,4 \%$ & $1,6 \%$ \\
\hline & $\%$ dentro de Origen & $13,7 \%$ & $36,4 \%$ & $41,4 \%$ & $14,3 \%$ \\
\hline & $\%$ del total & $9,7 \%$ & $8,1 \%$ & $1,9 \%$ &, $3 \%$ \\
\hline \multirow[t]{4}{*}{ Nunca } & Recuento & 187 & 59 & 11 & 8 \\
\hline & $\begin{array}{l}\% \text { dentro de Qué haces en tu } \\
\text { tiempo libre. Actividades } \\
\text { extraescolares }\end{array}$ & $70,6 \%$ & $22,3 \%$ & $4,2 \%$ & $3,0 \%$ \\
\hline & $\%$ dentro de Origen & $41,3 \%$ & $41,3 \%$ & $37,9 \%$ & $57,1 \%$ \\
\hline & $\%$ del total & $29,3 \%$ & $9,2 \%$ & $1,7 \%$ & $1,3 \%$ \\
\hline
\end{tabular}

Fuente: Elaboración propia a partir de los datos del estudio 
Hay una serie de razones que dificultan la convivencia entre navarros e inmigrantes, creando barreras entre unos y otros y manteniendo la sensación de formar grupos diferentes, basados en sentimientos de xenofobia. Se habla de que la sociedad tiene desconfianza frente a los inmigrantes por lo que se reconoce la existencia en la sociedad de xenofobia. Pero también hay conciencia de que los nativos son los primeros con los que se cuenta, por ejemplo, a la hora de dar un trabajo y se mantienen prejuicios de que los extranjeros, sobre todo hispanoamericanos, son poco fiables. Algunos creen que nos quitan el trabajo y se aprovechan de ventajas y servicios que no se dan a la población nativa. Incluso existen prejuicios de que los navarros pueden ser objeto de actos violentos por parte de los extranjeros, como de robo.

Los alumnos navarros participan mucho más que los inmigrantes en actividades extraescolares. El 81,7\% de los españoles participan a diario o algunos días en actividades escolares. Los hispanoamericanos lo hacen el 39,0\%, así como el 31,8\% de los alumnos de Europa del Este. El $67 \%$ de los magrebíes no participan. Estos datos son un indicio de un cierto aislamiento social de los inmigrantes.

No se puede hablar de que existen bandas estructuradas y organizadas en los centros y cuyo objetivo sea enfrentarse con otros colectivos. Pero sí que existen pandillas que marcan diferencias con otros grupos, que intervienen en peleas con cierta frecuencia y que ponen de relieve la existencia de una fractura real entre los hispanoamericanos y navarros.

\subsection{La perspectiva de los profesores, instituciones y otros agentes sociales}

Hay que reconocer la excelente labor que están realizando los centros educativos para atender las necesidades de los inmigrantes. Los programas de acogida están diseñados para ese primer momento de choque en que el alumno entra por primera vez en un centro de enseñanza de Navarra de manera que se siente aceptado y nada excluido al ingresar en el centro educativo. Sin embargo, una vez se ha realizado esa primera acogida parece que no están previstas actuaciones de integración a medio o largo plazo, porque se pone de manifiesto que no existe, de manera explícita, un seguimiento de cómo el alumnado se va a ir integrando o no en el centro y qué dificultades se va a encontrar. Los centros prefieren no dedicar una atención especial a los inmigrantes, sino tratarlos como cualquier alumno que en un momento determinado pueden necesitar de alguna ayuda. Por consiguiente no tienen diseñadas acciones específicas referidas a los inmigrantes, aunque de hecho, éstos sean los colectivos bien diferenciados que existen en el centro. Recalcan que las "acciones de acogida de cualquier alumno, de aquí y de fuera" son genéricas. A partir de la acogida piensan que la integración del alumno es un proceso que se produce de forma natural. Pero para los casos que no es así, debería existir un protocolo de actuación para hacerle frente, ya que sería un proceso que podría extenderse a lo largo de todo el curso.

La mayoría de los centros demandan, además de un plan académico para asignar niveles y superar déficit, un plan de atención individual para que los alumnos inmigrantes puedan superar el "período de duelo". Han dejado su país, con sus familiares, amigos, diversiones, valores y han llegado a un país donde no tienen amigos, apenas familia y solo algún paisano disperso en la ciudad.

Los centros constatan que mucho del alumnado inmigrante sufre una fuerte frustración que puede desencadenar en conductas agresivas hacia la propia sociedad receptora. Muchos alumnos extranjeros ven cómo viven aquí sus compañeros nativos, cómo disponen de dinero, las viviendas, las familias estructuradas, los trabajos diferentes, la duración de la jornada más 
regular. En este sentido la escuela puede provocarles reacciones de fuerte frustración y de "odio" hacia una sociedad de bienestar de la que ellos no pueden participar.

Pero no solo existen situaciones de frustración y de sentirse rechazados por parte de la sociedad de acogida, porque existen otras situaciones, en las que son los propios inmigrantes los que no quieren la integración rechazando algunas de las situaciones con las que se encuentran, como es el caso de los marroquíes, porque son muy reacios a la integración e incluso manifiestan resistencias evidentes, como es el no reconocimiento a profesoras o la utilización del velo. Este colectivo presenta muchas dificultades de integración: creen que con la educación se intenta asimilarlos culturalmente y que abandones sus convicciones, costumbres, tradiciones. No tienen especial interés en el estudio y su actitud muestra un elevado victimismo respecto de la actitud de los nativos hacia ellos. Es un hecho plenamente constatado que los padres, varones marroquíes no acuden cuando se les llama desde el centro, y vienen las madres que apenas hablan español. Sabemos también, según afirman algunos centros, que los alumnos matriculados en cursos inferiores al que les correspondería por su edad, manifiestan un rechazo a su situación por considerar que es un agravio, sin tener en cuenta los motivos reales que obligaron a tomar esa decisión, como es el nivel académico que presentaban los niños en el momento de la escolarización, situación que supone un problema psicológico que deberá ser atendido.

Las chicas adolescentes hispanoamericanas están expuestas a situaciones de fuertes conflictos internos que les pueden conducir a conductas no admitidas socialmente en España, como la fuga con algún chico, los embarazos no deseados o salidas con chicos mayores a centros de diversión lejos del pueblo donde viven. Tal como se deduce del análisis de las entrevistas, las niñas adolescentes hispanoamericanas tienen a su edad un proyecto inminente, aunque un tanto difuso, de matrimonio y de crianza de hijos. Están en edad de buscar pareja para la formación de una familia. De ahí su sorpresa de que las jóvenes nativas tengan los hijos en torno a los treinta años, porque a los quince años son "casaderas" y su actitud frente a la búsqueda de un chico que les guste les genera un grado mayor de dependencia. En sus países en esas edades son también mujeres que adquieren responsabilidades familiares, como el cuidado de hermanos menores o de personas mayores. En alguna entrevista se ha señalado que a los quince años las chicas dejan de ir a clase, porque asumen papeles responsables en su casa. Este planteamiento choca evidentemente con la cultura de las adolescentes de España porque no está en su horizonte asumir responsabilidades familiares, no piensan en el matrimonio, son más autónomas con respecto a los chicos, no piensan en tener hijos, tienen abiertas las relaciones sexuales y conocen los recursos para no quedarse embarazadas.

Existe también un riesgo para los programas de igualdad diseñado por los centros. El hecho de que las chicas, sobre todo sudamericanas, se muestren más atractivas para los chicos y más sumisas, en el marco del "machismo cultural" de sus países, ha provocado un efecto negativo en los programas de igualdad de género. Las chicas navarras están asumiendo un rol de mayor dependencia con respecto a los chicos, sobre todo cuando se relacionan con extranjeros.

Los jóvenes navarros no perciben a los inmigrantes como un problema que les afecta a ellos pero existe cierto miedo a que los inmigrantes sean un foco de violencia. Más en concreto tienen preocupación porque ciertos grupos, como los Lating Kings, "puedan crecer entre nosotros".

Los jóvenes navarros creen que existen diferencias profundas en base al estatus jurídico ("no somos iguales"), al origen cultural y a la situación económica. El fenotipo, en forma de 
rasgos físicos diferentes, sirve como refuerzo y protección de la propia identidad frente a los inmigrantes.

El estudio ha querido analizar si los jóvenes adolescentes que no están en contacto en las aulas y recreos con alumnos extranjeros manifiestan actitudes más o menos xenófobas que los alumnos que conviven con ellos en los centros. El estudio no evidencia la existencia de actitudes o prejuicios diferentes a los de los alumnos que estudian en centros con un porcentaje importante de inmigrantes.

Sobre sus posibilidades ante el futuro, todos los alumnos de los centros analizados reconocen la igualdad de todos y el derecho que tienen los inmigrantes a las mismas oportunidades que los autóctonos, aunque creen que por razones culturales, y de cierta situación de exclusión social, así como de no disponer de recursos, tendrán menos oportunidades de éxito que los alumnos nativos.

\section{CONCLUSIONES}

La conclusión principal que se desprende de este breve análisis es que se mantiene la hipótesis de que los inmigrantes se han ido identificando a sí mismos como alumnos distintos, y que han ido configurando su imaginario social paralelo al de los jóvenes autóctonos con los que conviven en las mismas aulas de los centros de enseñanza secundaria obligatoria.

Una vez confirmada este hipótesis una segunda mirada hace que nos demos cuenta que existen procesos de exclusión y rechazo a los jóvenes inmigrantes.

El centro educativo es espacio de convergencia entre alumnos autóctonos y extranjeros, pero no solo por este hecho se dará la integración, sino que tendrán que ponerse en práctica otra serie de factores que ayuden a un acercamiento entre los unos y los otros para formar el nosotros, para que realmente se dé la integración.

Muchos centros realizan maniobras de aproximación de ambas realidades, con mayor o menor grado de éxito, pero con la convicción de que el acercamiento facilita la aceptación mutua y la construcción conjunta de un imaginario social compartido.

\section{BIBLIOGRAFÍA}

AJA, E. y ARANGO, J. (2006). Veinte años de inmigración en España: perspectivas jurídica y sociológica. (1985-2004). Barcelona: Sociológicas. Colección Academia 28.

BARBOSA, F. (2007). Jóvenes e Inmigración en Madrid: espacios de sociabilidad. Madrid: Observatorio de las migraciones. Dirección General de Inmigración.

BESALU COSTA, X. (2002). Diversidad Cultural y educación. Madrid: Síntesis.

MASSOT, M. I. (2003). Jóvenes entre culturas. La construcción de la identidad en contextos culturales. Bilbao: Desclée de Brouwer.

PONCE, J. (2007). ¿Gueto escolares? Segregación escolar e inmigración. Derechos y políticas públicas urbanas. Madrid: CEC

PRÉVOS, G. (2004). "La integración de los jóvenes salidos de la inmigración magrebí en Francia”, en Revista de Estudios de Juventud, 66: 85-101.

TORREBLANCA, J. (2006). "Educación y emigración", en Temas para el debate, $\mathrm{n}^{\mathrm{o}} 136$

VALERO, J. R. (2002). Inmigración y escuela. La escolarización en España de los hijos de los inmigrantes. Alicante: Publicaciones de la Universidad de Alicante. 


\section{Breve currículo:}

\section{Neus Caparrós Civera}

Doctora en Ciencias de la Educación. Licenciada en Ciencia Políticas y Sociología, y Diplomada en Trabajo Social. Actualmente es profesora de Trabajo Social y Servicios Sociales en la Universidad de la Rioja. Ha participado en varios proyectos de investigación en temáticas relacionadas con la familia, los menores, la cooperación al desarrollo, las políticas sociales y la innovación social. Es miembro de la Cátedra Unesco de La Rioja.

\section{Esther Casares García}

Doctora en Sociología. Profesora Doctora de Sociología en la Universidad Pública de Navarra. Desarrolla su labor docente e investigadora en temas relacionados con familia, género y educación 